\title{
Discourse analysis
}

David F Marks

Potential competing interests: The author(s) declared that no potential competing interests exist.

Discourse analysis is a set of procedures for analysing language as used in speech or texts. 